\title{
Criminologie
}

\section{Le vol à main armée chez les mineurs}

\section{Marc LeBlanc}

Volume 18, numéro 2, 1985

Le vol à main armée à Montréal

URI : https://id.erudit.org/iderudit/017214ar

DOI : https://doi.org/10.7202/017214ar

Aller au sommaire du numéro

Éditeur(s)

Les Presses de l'Université de Montréal

ISSN

0316-0041 (imprimé)

1492-1367 (numérique)

Découvrir la revue

Citer cet article

LeBlanc, M. (1985). Le vol à main armée chez les mineurs. Criminologie, 18(2),

7-34. https://doi.org/10.7202/017214ar
Résumé de l'article

Armed robbery is rarely committed by minors, but because of the gravity of the crime, it is given considerable importance in the operations of our justice system. Since there is little data in the regard, we have investigated how such crimes, which are among the most serious, are treated by the system.

We have found that these crimes are committed in an unprofessional way. From the police to the defence procedure, and going through the director of youth protection, they are dealt with fairly rapidly and in an official manner; however, the sentence is based less on the objective gravity of the crimes than on the characteristics of the minor, on his previous criminal record. 
Armed robbery is rarely committed by minors, but because of the gravity of the crime, it is given considerable importance in the operations of our justice system. Since there is little data in the regard, we have investigated how such crimes, which are among the most serious, are treated by the system.

We have found that these crimes are committed in an unprofessional way. From the police to the defence procedure, and going through the director of youth protection, they are dealt with fairly rapidly and in an official manner; however, the sentence is based less on the objective gravity of the crimes than on the characteristics of the minor, on his previous criminal record.

Le vol à main armée apparaît, même chez les mineurs, comme l'aboutissement ultime de l'enracinement criminel à la fin de l'adolescence. Comme le démontrent Fréchette et LeBlanc (1979. 1985), vers seize ou dix-sept ans, cette forme de délinquance se commet par des jeunes qui ont déjà un passé criminel relativement long et lourd, en termes de variété, de volume et de gravité des délits déjà commis. C'est un aboutissement. La description des voleurs à main armée d'âge mineur de Montréal a déjà été faite par Bruneau et al. (1981). Dans le cadre de notre programme de recherche sur le vol à main armée, nous avons eu l'occasion d'approfondir la connaissance de cette forme de criminalité chez. les mineurs et, plus particulièrement, son cheminement dans le système de justice pour mineurs : la façon dont la police, la Direction de la protection de la jeunesse (DPJ) et le tribunal y répondent en appliquant la Loi sur les jeunes délinquants et la Loi de la protection de la jeunesse.

Ceci étant dit, rappelons que la police a poursuivi 154 mineurs pour avoir été impliqués dans un vol à main armée. Il s'agit d'un échantillon des vols à main armée à Montréal en 1981 et à Québec en 1980-1981 (voir Gagnon et LeBlanc, 1984). Au niveau de la Direction de la protection de la jeunesse, nous sommes arrivés à

* Cette recherche a été réalisée grâce à une subvention triennale de la Fondation FCAR et une contribution de l'Université de Montréal.

** Professeur titulaire, École de criminologie et chercheur associé. Centre international de criminologie comparée, Université de Montréal. 
retracer 125 dossiers de ces jeunes dans les centres des services sociaux de Québec et de Montréal. Pour ce qui est des 29 dossiers manquants, il faut préciser que nous nous sommes limités aux signalements acheminés aux centres des services sociaux de Montréal et de Québec : pour la région de Montréal particulièrement, certains jeunes bien qu'ayant commis leur délit sur le territoire de la Communauté urbaine relèvent de centres des services sociaux extérieurs et leurs dossiers par conséquent ne se retrouvent pas au lieu même du délit. Ajoutons enfin que dans quelques cas nous n'avons pu retracer le dossier du jeune puisqu'il était en circulation auprès des praticiens au moment de notre étude.

\section{PLAINTES FORMULÉES PAR LA POLICE ET SIGNA- LEMENTS REÇUS À LA D.P.J.}

\subsection{TYPOLOGIE E TDESCRIPTIONS DES VOLS A MAIN ARMÉE IMPLIQUANT DES MINEURS}

Avant d'entreprendre la description des jeunes à travers les dossiers de la D.P.J. et ceux du tribunal de la jeunesse, il convient de préciser dans quels genres d'événements ils sont impliqués. Participent-ils aux mêmes types de vols à main armée que les adultes ou les retrouve-t-on plutôt dans certaines catégories? Le tableau 1 permet de répondre à cette question.

\section{TABLEAU 1}

Types de vols à main armée, mineurs signalés d̀ la D.P.J. et adultes poursuivis au tribunal : comparaisons

Types de vol à main armée

Mineurs signalés Adultes poursuivis $\mathbf{N}=\mathbf{1 2 5}$ $\mathbf{N}=\mathbf{5 1 5}$

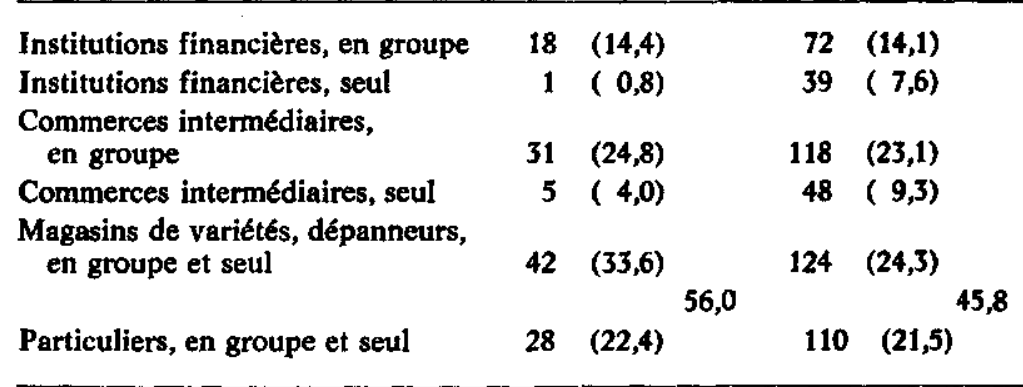


Les mineurs signalés à la D.P.J. sont impliqués dans des vols à main armée comparables à ceux commis par les adultes exception faite qu'ils figurent beaucoup moins souvent dans les types deux et quatre, c'est-à-dire les vols commis seul contre les institutions financières et les commerces intermédiaires. Les mineurs sont presque absents de ces catégories alors qu'ils se retrouvent dans une proportion plus importante que les adultes dans la catégorie des vols à main armée dirigés contre les magasins de variété et les dépanneurs $(33,6 \%$ contre $24,3 \%)$. Qu'il s'agisse d'un type de vol ou d'un autre, une constante demeure : les mineurs rapportés à la D.P.J. ont rarement exécuté seul leur vol à main armée. En effet, ce n'est que $13,6 \%$ de ces derniers qui commettent seul leur délit alors que pour les adultes traduits devant le tribunal cette proportion s'élève à $29,3 \%$. Il ressort que les voleurs à main armée âgés de moins de 18 ans opèrent plus souvent en groupe, du moins cela est vrai en ce qui concerne les dossiers solutionnés. En ce qui a trait à l'ensemble des événements signalés à la police et figurant dans notre échantillon, les descriptions faites par les témoins et victimes font état des mêmes tendances (Gagnon et LeBlanc, 1984).

Quant à savoir si le vol est réalisé avec une arme à feu ou une autre arme offensive, nos données font voir que $51,3 \%$ des mineurs signalés sont impliqués dans un vol commis avec une arme à feu et $48,7 \%$ avec une autre arme offensive ${ }^{1}$. Du côté adulte, ces proportions sont de $70,1 \%$ et de $29,9 \%$. Cette différence apparaît majeure en ceci qu'elle signale que les vols à main armée impliquant des mineurs sont moins organisés et planifiés. Cette caractéristique s'applique aussi à l'ensemble des vols à main armée (Gagnon et LeBlanc, 1984). Chez les mineurs, cet «amateurisme» est en quelque sorte plus prononcé. Il fait référence aux moyens dont disposent le ou les suspects, ces éléments déterminant dans une certaine mesure les victimes potentielles. En effet, on peut penser que les mineurs ont moins de possibilités (contacts, moyens financiers,...) de se procurer une arme à feu

1. Ces chiffres ne disent pas si le mineur a une arme à feu ou une autre arme offensive pour commettre le vol mais plutôt ce qui a été vu par les témoins. Le suspect le plus en vue peut par exemple avoir un revolver et l'autre un couteau ou pas d'arme du tout. Il nous est impossible de préciser ces nuances pour la raison très simple que les suspects décrits n'ont pas de nom! 
ou encore qu'ils participent à des délits nécessitant moins souvent une arme à feu. D'une part, ils sont impliqués dans des délits commis contre les dépanneurs et les particuliers dans $56 \%$ des cas contre $45,8 \%$ au niveau adulte et d'autre part, ils sont rarement seuls; ces deux types d'informations permettent de comprendre pourquoi on les retrouve moins nombreux dans la catégorie des vols à main armée commis avec arme à feu.

\subsection{CONCORDANCE OU DISCORDANCE ENTRE PLAINTES ET SIGNALEMENTS}

Au niveau adulte, nous avons établi (Gagnon et LeBlanc, 1984) qu'entre la formulation des plaintes policières et les accusations formulées par le tribunal il n'existe pas de décalage important, c'est-à-dire que la poursuite s'en tient de façon générale aux chefs allégués par les corps policiers. Au niveau des mineurs, il ne sera pas question de chefs d'accusation dans un premier temps puisque les signalements de la police sont acheminés à la D.P.J. qui en soi n'est pas une instance judiciaire. À ce stade, il sera donc question de motif de signalement; pour notre cueillette de données, nous avons retenu les motifs tels qu'ils sont définis au niveau des formulaires de cet organisme. Pour cette raison, par conséquent, l'analyse de la concordance doit se faire entre chefs et motifs.

Pour l'ensemble des cas (tableau 2), nous observons que $69,6 \%$ des mineurs signalés à da D.P.J. le sont pour un article 302D (un vol avec arme à feu) aux dires de la police alors que $\mathbf{2 2 , 5} \%$ le sont en vertu d'un article 302 sans aucune précision de l'alinéa (et ainsi de la notion de l'arme). Cette difficulté à préciser la nature du vol qualifié avait déjà été soulevée au niveau de l'ensemble des personnes poursuivies par la police; nous observions en effet, que pour $15,9 \%$ des 1177 accusés, les policiers ne mentionnaient qu'un article 302 dans leur demande d'intenter des procédures, sans précision supplémentaire. Pour les 125 mineurs signalés à la D.P.J. cette proposition s'élève à $22,4 \%$, ce qui nous amène à penser que cette imprécision est plus fréquente à leur égard que ce n'est le cas pour les adultes.

Au niveau de la concordance entre police et D.P.J., on note que dans $67,8 \%$ des cas où la police allègue un article 302D, la D.P.J. fait référence à un vol qualifié commis avec une arme (deux premières catégories) ce qui veut dire que dans un peu plus 
TABLEAU 2

Concordance ou discordance entre le chef principal au niveau de la police et motif de référence da la D.P.J.

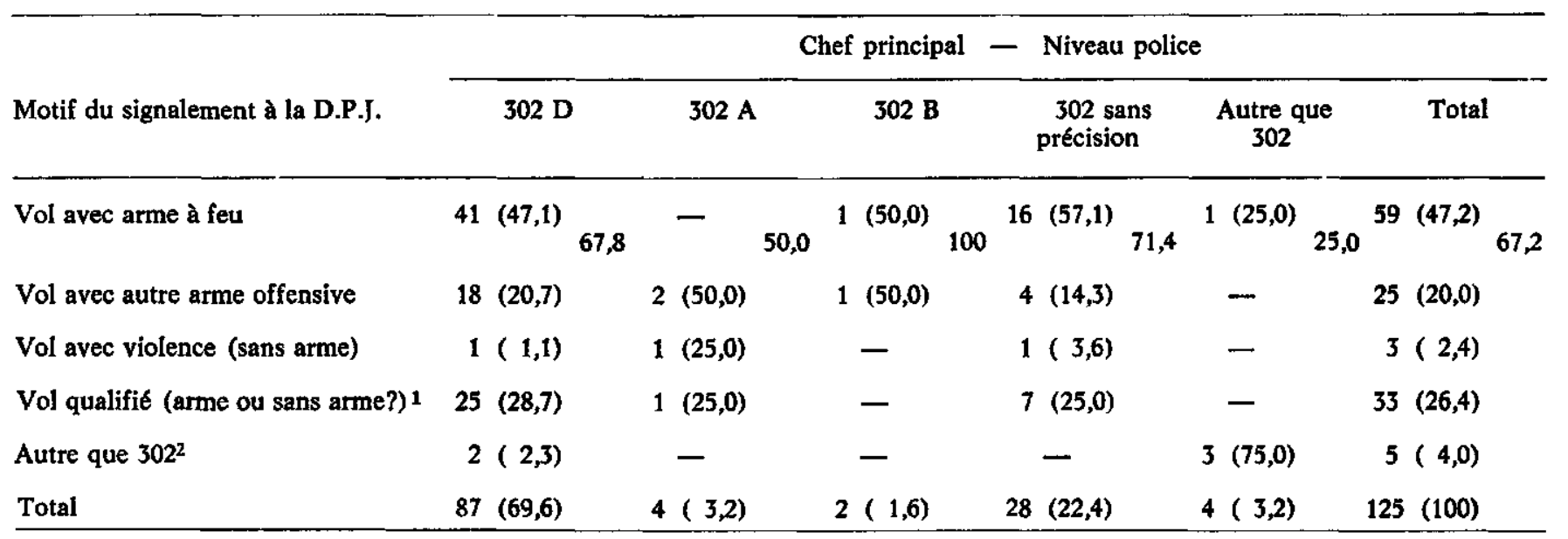

1. Dans cette catégorie le type du vol qualifié n'est pas précisé, c'est-à-dire qu'on ne peut pas dire s'il s'agit d'un vol qualifié avec ou sans arme.

2. Les dossiers indiquaient un vol qualifié mais par la suite la demande d'intenter des procédures a été modifiée à notre insu. 
de deux cas sur trois il y a concordance parfaite. Toutefois, pour $28,7 \%$ des cas l'article 302D, la D.P.J. parle plutôt d'un motif de vol qualifié, sans préciser s'il y a ou non une arme offensive. Pour ces cas donc, la demande d'intenter des procédures de la police est ajustée en fonction de la version de l'individu. D'un point de vue strictement juridique, un individu impliqué dans un vol à main armée, qu'il ait ou non lui-même une arme lors du délit, sera poursuivi en vertu d'un article 302D. Au niveau de la D.P.J.. on peut penser que si l'événement a une certaine garantie, la contribution du jeune ou si on veut sa participation réelle dans l'événement revêt un caractère tout aussi important. Dans ce sens, il est possible qu'au niveau du motif de signalement on tienne compte du rôle du jeune, ce qui serait susceptible d'expliquer ce décalage.

Lorsque nous étudions maintenant les 28 cas pour lesquels la police n'avait pas précisé la nature du vol qualifié, la D.P.J. classe $71,4 \%$ de ces cas dans les catégories de vol avec arme offensive ce qui nous fait voir que l'événement est analysé pour ce qu'il est. Ces décalages entre le niveau de police et celui de la D.P.J. n'auront d'impact réel que dans la mesure où nous observerons que les décisions conjointes sont fonction des motifs de signalement; dans le cas où il n'y a pas de relation à ce niveau ce sont les chefs d'accusation au tribunal de la jeunesse qu'il faudra étudier en fonction des demandes d'intenter des procédures.

\section{LES SIGNALEMENTS REÇUS A LA D.P.J.} ET LEUR TRAITEMENT

\subsection{NOMBRE ET NATURE DES ÉVÉNEMENTS}

Lorsqu'un mineur est relié à un délit, il peut être signalé à la D.P.J. pour ce motif ainsi que pour d'autres infractions s'il y a lieu; si plusieurs événements sont rapportés séparément mais dans un délai relativement court, ils peuvent tous être inclus dans le même signalement. Pour les mineurs impliqués dans au moins un des vols à main armée faisant partie de la présente étude, le contenu des signalements va de un à vingt-huit (28) événements.

Pour un peu moins de la moitié des cas, un seul événement est rapporté à la D.P.J. ${ }^{2}$ : cela signifie que la décision conjointe ne portera que sur le vol à main armée à l'étude. Pour le quart des cas toutefois, il est intéressant de voir que quatre événements ou 
plus figurent dans le signalement : ceci laisse à penser que certains mineurs ont une délinquance numériquement importante. En fait, pour plus de la moitié des cas $(53,7 \%)$ deux délits ou davantage sont inscrits dans le signalement. Cette information n'est pas sans intérêt en ce qui a trait à la carrière criminelle de certains jeunes; il est évident que ces délits peuvent être condensés dans un temps relativement court mais cela donne néanmoins une indication quant au degré d'implication dans les activités délictuelles. Par l'étude de la nature de tous ces événements nous aurons une idée de la part du vol à main armée dans la criminalité juvénile (tableau 3).

\section{TABLEAU 3}

Nature des événements rapportés dans le signalement da la D.P.J.

\begin{tabular}{|c|c|c|c|c|c|c|}
\hline \multirow[t]{2}{*}{ Nature des événements } & \multicolumn{3}{|c|}{$\begin{array}{c}\text { Ensemble des délits } \\
\text { dans le signalement }\end{array}$} & \multicolumn{3}{|c|}{ Délits à l'étude } \\
\hline & $\mathrm{N}$ & $\%$ & $\begin{array}{c}\% \\
\text { cumulatif }\end{array}$ & $\mathbf{N}$ & $\%$ & $\begin{array}{c}\% \\
\text { cumulatif }\end{array}$ \\
\hline Vol avec arme à feu & 127 & 35,3 & 35,3 & 59 & 47,2 & 47,2 \\
\hline $\begin{array}{l}\text { Vol avec autre arme } \\
\text { offensive }\end{array}$ & 56 & 15,5 & 50,7 & 26 & 20,0 & 67,2 \\
\hline $\begin{array}{l}\text { Vol qualifié } \\
\text { (arme ou sans arme) }\end{array}$ & 93 & 25,7 & 76,4 & 33 & 26,4 & 93,2 \\
\hline $\begin{array}{l}\text { Vol avec violence } \\
\text { (sans arme) }\end{array}$ & 10 & 2,7 & 79,1 & 3 & 2,4 & 96,0 \\
\hline Vol par effraction & 22 & 6,2 & 85,2 & - & - & - \\
\hline Vol par effraction et recel & 29 & 8,0 & 93,2 & - & - & - \\
\hline Autres & 24 & 6,6 & 99,8 & 5 & 4,0 & 100,0 \\
\hline Total & $361^{1}$ & 100,0 & - & 125 & 100,0 & - \\
\hline
\end{tabular}

1. Pour 43 délits nous n'avons pas l'information.

Si nous regardons de près le contenu des signalements, nous observons d'abord que pour $96 \%$ des dossiers étudiés, le motif

2. Au niveau du tribunal adulte, nous avions vu que $64,3 \%$ des 515 personnes poursuivies ne l'étaient que pour le vol à main armée à l'étude, ce qui est supérieur à la donnée pour les mineurs. Les adultes sont poursuivis pour une moyenne de 2,10 événements avec une médiane de 1,28. Les mineurs sont donc associés à plus de délits que les adultes lorsqu'ils sont arrêtés. 
en est un de vol qualifié en ce qui a trait au délit à l'étude $(\mathrm{N}=125)$; dans $47,2 \%$ on précise que le vol a été commis avec une arme à feu, dans $20 \%$ avec une arme offensive d'un autre type et enfin dans $26,4 \%$ des cas, il n'est fait mention d'aucune sorte d'arme. Il est intéressant de noter, parallèlement à cela, que $79,1 \%$ de l'ensemble des délits contenus dans les signalements sont aussi des vols qualifiés ce qui indique qu'il y a concentration, du moins dans un laps de temps restreint, dans un agir délinquant particulier. Nous ne disons pas que ces jeunes ne sont pas impliqués dans d'autres formes d'infractions, mais plutôt que pour la délinquance connue, le vol qualifié occupe une place prépondérante. Quant au vol à main armée en particulier, il rend compte de $50,7 \%$ des 361 délits et il faut préciser que dans $25,7 \%$ des cas, il s'agit d'un vol sans mention quant au recours à une arme, auquel cas la proportion des vols à main armée serait plus grande encore. Il ne fait donc nul doute que pour certains jeunes cette forme de criminalité est particulièrement importante.

\subsection{QUI SONT LES MINEURS SIGNALÉS À LA D.P.J. POUR VOL A MAIN ARMÉE?}

Pour ce qui est du signalement qui retient notre attention, nous avons vu que les délits impliqués se concentrent dans la catégorie des vols qualifiés. Lorsque nous regardons leur passé, il ressort, d'abord, que 28 jeunes sont totalement inconnus des agences, c'est-à-dire qu'ils n'ont jamais été signalés à la D.P.J. depuis l'adoption de la Loi 24 en janvier 1979 et qu'ils n'ont jamais comparu devant la Cour de Bien-Être social avant cette date. Il y a 48 mineurs, soit $38,4 \%$ qui ont déjà comparu devant la cour avant janvier 1979 d'une part et qui ont été l'objet d'un signalement à la D.P.J. depuis janvier 1979 d'autre part; également, il y a 42 mineurs, soit $33,6 \%$ pour lesquels on ne relève aucune comparution avant janvier 1979 mais au moins un autre signalement que celui à l'étude depuis janvier 1979 mais n'ayant jamais été signalés depuis l'adoption de la Loi 24.

Ces chiffres permettent d'établir que près de $80 \%(77,6)$ des mineurs signalés pour un vol à main armée sont déjà connus que ce soit de la Cour de Bien-Être social avant 1979 ou de la D.P.J. après cette date. Si nous concentrons notre analyse sur la période débutant en janvier 1979, et pour laquelle nous avons des informations plus détaillées, nous observons que 90 des 125 
mineurs ont déjà été signalés à la D.P.J. au cours de cette période, soit une proportion de $72 \%$. Bruneau et al. (1981) rapportaient pour leur part que les deux tiers de leur échantillon avaient des antécédents criminels.

Le nombre de signalements antérieurs au délit, pour cette période relativement courte ${ }^{3}$ va de un à 24 pour une moyenne de 4,97. Non seulement la proportion de jeunes ayant des signalements antérieurs depuis 1979 est-elle élevée mais le nombre de ces signalements l'est également. En effet, nous constatons que 28,1\% des jeunes en ont six ou davantage. Si d'un côté il y a $28 \%$ de ces jeunes qui en sont à leur premier signalement depuis janvier 1979 il y en a par ailleurs près de $30 \%$ qui présentent une histoire sociale chargée.

Les motifs de ces signalements seront soit la délinquance (article 40), soit la protection (article 38). Le tableau 4 donne un aperçu des tendances observées. Il y a donc près de $85 \%$ des mineurs déjà signalés à la D.P.J. depuis 1979 qui l'ont été pour au moins un article 40 , et $53,3 \%$ pour au moins un article 38 . Cette donnée indique qu'une proportion très importante de jeunes ont déjà été rapportés, depuis janvier 1979, pour un motif de délinquance. Tous les signalements antérieurs depuis janvier 79 sont pour délinquance dans une proportion de $46,6 \%$. Si nous analysons la nature des délits impliqués dans ces signalements, nous notons qu'il y a $36,7 \%$ de tous les mineurs rapportés au moins une fois à la D.P.J. depuis janvier 1979 qui l'ont été pour au moins un vol qualifié. Cela revient à dire que plus du tiers des jeunes ayant déjà été l'objet d'un signalement à la D.P.J. avaient été impliqués dans un vol qualifié. Par ailleurs, les délits contre la propriété figurent parmi les plus répandus chez ces jeunes; leur carrière depuis 1979 semble se concentrer sur les délits contre les biens et le vol qualifié.

3. Entre le vol à main armée et le signalement antérieur le plus vieux depuis janvier 1979, la durée moyenne de cette période est de 292 jours avec une médiane de $254,5(\mathrm{~N}=86)$. Cela revient à dire que pour l'ensemble des mineurs signalés à la DPJ depuis janvier 1979, il y a en moyenne 9,7 mois entre le vol à l'étude et le signalement le plus ancien depuis janvier 1979. 
TABLEAU 4

Motifs des signalements antérieurs depuis janvier 1979 (non cumulatif)

Motif du ou des signalements antérieurs (janvier 1979)

$\mathrm{N} \%(\mathrm{~N}=90)$

Au moins un signalement antérieur pour délinquance (article 40) depuis janvier 1979

$76 \quad 84,4$

Au moins un signalement antérieur pour protection (article 38) depuis janvier 1979

$48 \quad 53,3$

Tous les signalements antérieurs depuis janvier 1979 sont pour délinquance (article 40)

$42 \quad 46,6$

Tous les signalements antérieurs depuis janvier 1979 sont pour protection (article 38 )

$14 \quad 15,5$

Les données de la présente section témoignent de l'implication de ces jeunes dans une délinquance relativement abondante, c'était aussi le cas pour la délinquance cachée (Fréchette et LeBlanc, 1979, 1985; Bellot et LeBlanc, 1984) ; la présentation que nous venons de faire rend compte d'un minimum de conduites délictuelles parce que nos informations détaillées se limitent à la période débutant en janvier 1979 d'une part et qu'il s'agit de comportements connus d'autre part. Retenons que les mineurs impliqués dans les vols à main armée de notre étude sont connus des agences officielles dans plus de $75 \%$ des cas et que $60,8 \%$ $(76 / 125)$ de ces jeunes ont déjà été rapportés à la Direction de la protection de la jeunesse pour un article 40 depuis janvier 1979. Il ne semble donc pas que le vol à main armée constitue une première dans la «carrière criminelle» de nos jeunes voleurs à main armée. Nous ajouterons à ces descriptions que pour les 90 jeunes ayant des signalements antérieurs à la Direction de la protection de la jeunesse, il y en a $74,5 \%$ pour lesquels au moins un signalement antérieur a déjà été judiciarisé. Chaque jeune déjà signalé $(\mathrm{N}=90)$ a vu en moyenne 2,91 signalements donner lieu à une judiciarisation. Cet aspect de la question rend compte de la gravité des signalements antérieurs. Non seulement plusieurs jeunes sont-ils connus de la D.P.J. depuis 1979 mais plusieurs le sont aussi du tribunal de la jeunesse. Ajoutons à cela que 48,8 \% de ces jeunes faisant partie de notre échantillon $(61 / 125)$ ont déjà été confiés pour une période déterminée à un centre d'accueil 
depuis janvier 1979. Le nombre de ces placements varie de 1 à 5 pour une moyenne de 2,03 par individu. Tout cela permet de n'en plus douter : ces jeunes sont connus des agences officielles, ils ont à leur compte des comportements délinquants nombreux et ils ont donné lieu à des interventions sociales et judiciaires antérieures au vol à main armée.

Pour terminer le profil descriptif de ces jeunes, voyons leur âge. Les pourcentages font voir que $61,2 \%$ de ces jeunes ont plus de 16 ans et demi et $82,9 \%$ ont plus de 15 ans et demi. La moyenne s'élève à 16,6 ans (donnée confirmée par Bruneau et al., 1984). À propos du sexe de ces jeunes, précisons que quatre mineurs signalés à la D.P.J. sont de sexe féminin pour une proportion de 3,2\%. Au niveau de la police, six mineurs avaient été recensés; un cas relevait d'un CSS extérieur et un cas était impossible à suivre parce que nous n'avons pas le nom de l'accusé.

\subsection{LA DÉCISION CONIOINTE DANS LES CAS DE VOL À MAIN ARMÉE}

D'une manière générale, il semble y avoir une consigne à l'effet de judiciariser le cas des vols à main armée puisque $90,4 \%$ des 125 jeunes l'ont été. Pour cinq cas, soit $4 \%$ des jeunes, des mesures volontaires furent adoptées et sept dossiers ont été fermés, soit $5,6 \%$. La première question qui se pose est de vérifier en quoi les douze cas non judiciarisés sont différents de l'ensemble des cas; nous avons fait cette étude pour les variables les plus pertinentes.

Au niveau des données de la police, nous observons que les jeunes qui ne furent pas judiciarisés sont impliqués plus souvent que les autres dans des vols à main armée des types cinq et six, c'est-à-dire un vol commis contre les commerces de variétés, dépanneurs ou contre les particuliers. Aucun des mineurs déjudiciarisés n'avait commis son méfait contre une institution financière. Quant au chef principal tel que formulé à la police, pour $66,7 \%$ des cas il s'agit d'un article 302 sans précision de l'alinéa alors que pour l'ensemble des cas, cette proportion est de $22,2 \%$. Par ailleurs, c'est dans une proportion plus importante également que le chef ne porte pas sur l'article $302(16,7 \%$ contre $3,2 \%)$. Ces données suggèrent que les mineurs déjudiciarisés sont impliqués dans des délits où il n'était pas manifeste qu'il s'agissait d'un 
vol à main armée. Dans ces cas, on peut se demander s'il y avait effectivement une arme, s'il y avait violence et par conséquent s'il s'agissait d'un vol qualifié ou d'un vol simple. Vu la cible également, on peut penser que la dynamique du délit n'était pas caractéristique du vol à main armée; nous avons déjà vu que pour les types cinq et six le chef principal était plus difficile à qualifier pour les policiers. Nous remarquons également qu'au niveau de la D.P.J. le motif du signalement n'est pas un vol qualifié dans une proportion plus grande que pour l'ensemble des cas $(16,6 \%$ contre $2,6 \%$ ).

Nous avons aussi analysé le contenu du signalement, il y a beaucoup moins d'événements en cause; dans $75 \%$ des cas non judiciarisés le vol à main armée est le seul délit contre $46,3 \%$ pour l'ensemble des 125 mineurs. Au chapitre des antécédents, les jeunes qui ne sont pas judiciarisés présentent une feuille de route nettement moins chargée en ce qui a trait à la délinquance; par contre, ils ont été signalés plus souvent que les autres pour un motif de protection. Il ressort que lorsque le cas est connu, il l'est essentiellement pour un motif de protection. Quant à l'âge, il est comparable entre les groupes bien que les cas non judiciarisés soient légèrement plus jeunes $(16,16$ contre 16,60$)$. Ajoutons à ces résultats qu'une seule fille parmi les quatre de notre échantillon n'a pas été judiciarisée; cela représente une proportion de $25 \%$ contre $9,6 \%$ pour l'ensemble des sujets.

D'une manière générale, nous observons que les mineurs qui ne sont pas judiciarisés sont impliqués dans un vol à main armée dont la gravité n'est pas évidente si on considère le chef principal formulé par la police. Par ailleurs, le signalement n'a pas l'ampleur des autres cas quant au nombre d'événements. Il ressort également que les jeunes qui ne sont pas traduits devant le tribunal ont moins d'antécédents pour délinquance et davantage pour protection. On peut penser que leurs gestes délinquants sont davantage inscrits dans une dynamique liée à leur situation de protection plutôt qu'associés à une carrière criminelle.

En dernier lieu, précisons que pour $88,3 \%$ des cas judiciarisés, ce sont tous les événements contenus dans le signalement qui ont été judiciarisés ce qui revient à dire qu'il est rare que la D.P.J. retire des délits de la plainte telle que formulée par les policiers. Voyons maintenant ce qu'il advient des jeunes traduits devant le juge. 


\section{LES CAS IUDICIARISÉS : INTERVENTION DU TRIBUNAL}

Le nombre de cas judiciarisés s'élève à 110 . Précisons que parmi ces cas il y en a quelques-uns pour lesquels nous n'avions pas retrouvé le dossier à la D.P.J. Dans la présente section nous chercherons à vérifier si les chefs d'accusation formulés devant le tribunal de la jeunesse coïncident avec ceux dont fait état la police, quel est le contenu et l'ampleur des dossiers judiciarisés et enfin quelles mesures sont prises par les juges à l'égard des mineurs impliqués dans le vol à main armée.

\subsection{CORRESPONDANCE OU DISCORDANCE ENTRE PLAINTES POLICIEERES ET CHEFS RETENUS AU TRIBUNAL DE LA JEUNESSE}

Tout d'abord nous observons que $88,1 \%$ des mineurs sont traduits devant le tribunal pour un article $302 \mathrm{D}$ du code criminel; au niveau de la police cette proportion était de $70,9 \%$, mais il faut se rappeler que pour les jeunes la proportion de cas où on ne précise pas l'alinéa est élevée $(20,9 \%)$. On remarque également que dans cinq cas, le tribunal identifie une tentative de vol à main armée alors que la police n'y fait jamais référence. Quant à la correspondance entre ces deux instances, on constate que $93,5 \%$ des chefs de 302D à la police sont ainsi maintenus au tribunal; les cinq cas qui n'ont pas conservé cet article ont été transformés en tentative $(302 \mathrm{C})$. Par ailleurs, $80 \%$ des mineurs poursuivis pour un article $302 \mathrm{~A}$ conservent cette étiquette au niveau du tribunal. En ce qui a trait au chef principal on peut donc dire que la correspondance est à peu de chose près parfaite. Au niveau des chefs secondaires on constate que le tribunal de la jeunesse conserve les chefs formulés par la police dans une proportion importante et que, par ailleurs, il ajoute souvent des chefs d'accusation (voir Gagnon et LeBlanc, 1984).

Ces résultats sont importants croyons-nous car ils viennent nous assurer d'une sorte de continuité entre les corps policiers et le substitut du procureur général, ce dernier ne venant pas réduire la gravité de l'offense par l'élimination de certains chefs secondaires ou la modification des chefs principaux. Ce sont les articles 83 (utilisation d'arme à feu) et 309 (déguisement) qui ne sont pas retenus dans quelques cas au niveau du tribunal. Les diffé- 
rences sont toutefois minimes. Le complot et le recel ${ }^{4}$ viennent en revanche aggraver les accusations. Si nous considérons le nombre de chefs reprochés par la police et par le procureur nous constatons que dans $72,7 \%$ des cas la concordance est parfaite; pour $20,9 \%$ des jeunes, le nombre des chefs est plus élevé au tribunal qu'à la police et dans à peine $6 \%$ des cas, il est inférieur.

\subsection{CONTENU DES DOSSIERS SOUMIS AU IUGE}

Comme nous l'avons déjà souligné, il est possible que plus d'un délit soit porté à l'attention du juge; il aura donc à décider d'une mesure en fonction de tous les événements rapportés et pas seulement pour un vol à main armée. Dans un premier temps, nous observons que les jeunes à qui on ne reproche qu'une seule infraction sont minoritaires. Pour le tiers des mineurs, il y a deux ou trois délits alors que pour un peu moins du quart il y en a quatre ou davantage; en moyenne chaque jeune se présente devant le juge pour un peu plus de trois délits $(3,18)$. Il va de soi que cela correspond à plusieurs chefs d'accusation. La distribution va de 1 à 47 pour une moyenne de 6,5 chefs par individu.

Au chapitre de la nature des chefs compris dans l'ensemble des dossiers, on observe d'abord que $45,4 \%$ des mineurs signalés pour le vol à main armée de notre étude sont également traduits devant le tribunal pour au moins un autre vol à main armée, ce qui n'est pas négligeable comme proportion. Quant aux chefs secondaires liés au vol à main armée le complot et le recel sont parmi les plus utilisés. Ce qui semble certain à la lumière de ces données c'est que les juvéniles qui sont poursuivis pour un vol à main armée le sont souvent pour une autre infraction et que cette dernière est un autre vol à main armée pour la moitié des cas.

Pour le vol à main armée à l'étude, les mineurs du tribunal se répartissent dans les divers types de vols à main armée d'une manière comparable aux mineurs rapportés à la D.P.J. (tableau 5 et 1). Ainsi, par rapport aux adultes poursuivis, les mineurs qui comparaissent devant le tribunal commettent rarement leur vol à main armée seuls et s'attaquent aux types cinq et six de manière prédominante.

4. Maintenant, il faut plutôt parler d'avoir en sa possession les biens obtenus illégalement, toutefois les intervenants utilisent le terme vol. 
TABLEAU 5

Typologie des vols à main armée pour les mineurs traduits devant le tribunal de la jeunesse

\begin{tabular}{lrr} 
Types de vols à main armée & $\mathrm{N}$ & $\%$ \\
\hline Institutions financières, groupe & 14 & 12,7 \\
Institutions financières, seul & 1 & 0,9 \\
Commerces intermédiaires, groupe & 30 & 27,3 \\
Commerces intermédiaires, seul & 4 & 3,6 \\
Variétés, dépanneurs, groupe et seul & 35 & 31,8 \\
Particuliers, groupe et seul & 26 & 23,6
\end{tabular}

\subsection{LES PLAIDOYERS ET VERDICTS}

D'abord il faut préciser qu'il y a huit jeunes pour lesquels le tribunal n'aura pas à statuer : cinq sont déférés au tribunal et pour trois autres le juge n'a pas juridiction pour procéder. Il reste done 102 cas sur lesquels portera notre analyse. Juste avant d'étudier ces cas, voyons sommairement les caractéristiques personnelles et délictuelles des individus qui ont été déférés.

Parmi les mineurs renvoyés au tribunal, trois ont dix-huit ans, un a dix-sept ans et pour un autre nous n'avons pas l'information. Deux de ces jeunes sont impliqués dans un vol de banque, deux dans un vol de commerce intermédiaire en groupe et un contre ce même type de cible mais seul. Trois de ces mineurs sont amenés devant le juge relativement à deux événements, un pour quinze et un pour dix-sept. En ce qui a trait aux cibles du vol à main armée et au nombre d'événements, on peut dire que ce sont des cas "sérieux». À propos des antécédents, tous ces mineurs ont déjà été signalés à la D.P.J. depuis janvier 1979 et quatre d'entre eux ont déjà été placés en centre d'accueil. Il est difficile, sur la base de ces seules données, de comprendre le pourquoi du déféré : nous savons que ces jeunes ont des antécédents et qu'ils ont déjà été l'objet d'une mesure de placement. En principe, le déféré ne peut survenir que si la preuve est établie à l'effet que toutes les mesures ont déjà été essayées et qu'en dépit de cela, le jeune poursuit son implication dans la carrière criminelle. Une chose est certaine : 
cette mesure est exceptionnelle $(4,5 \%)$ et il y a tout lieu de croire qu'on y a pas recours de manière abusive.

Bien que les procédures au niveau juvénile ne sont pas reconnues pour être aussi rigoureuses qu'au niveau adulte, les mineurs doivent quand même enregistrer un plaidoyer pour chacun des chefs pour lesquels ils sont accusés. Une étude chef par chef nous renseigne sur le fait que certains chefs sont plus souvent retirés alors que pour d'autres, on a tendance à plaider coupable (tableau 6).

TABLEAU 6

Nature des plaidoyers selon certains chefs pour l'ensemble de la dénonciation

\begin{tabular}{lccccc}
\hline & $\begin{array}{c}\text { Total des } \\
\text { chefs dans } \\
\text { les dénon- } \\
\text { ciations }\end{array}$ & $\begin{array}{c}\text { Plaide } \\
\text { couple } \\
\text { Article }\end{array}$ & $\begin{array}{c}\text { Chef } \\
\text { retiré } \\
\%\end{array}$ & $\begin{array}{c}\text { Chef } \\
\text { acquitté } \\
\%\end{array}$ & $\begin{array}{c}\text { Autre } \\
\%\end{array}$ \\
\hline 302 D & 202 & 83,2 & 1,9 & 1,9 & 13,0 \\
423 (complot) & 96 & 22,9 & 51,0 & - & 26,1 \\
83 (utilisation & 13 & 53,8 & 23,0 & - & 23,2 \\
$\quad \begin{array}{l}\text { arme à feu } \\
309 \text { (déguisement) }\end{array}$ & 28 & 60,7 & 14,2 & - & 25,1 \\
312 (recel) & 85 & 17,6 & 80,0 & - & 2,4 \\
\hline
\end{tabular}

Pour $83,2 \%$ des 202 articles 302 D figurant dans les dossiers des mineurs, un plaidoyer de culpabilité est enregistré. Le complot est retiré dans plus de la moitié des fois où il est formulé; on plaide coupable sur l'article 83 dans plus de la moitié des cas mais cela est sans conséquence grave puisque cela ne commande pas une peine minimale et consécutive d'un an comme du côté adulte. Au déguisement, on plaide coupable sur 60,75 des chefs alors que le recel est retiré pour $80 \%$ des cas où il est reproché. Nous avions vu que le chef de recel avait non seulement été conservé par le tribunal mais que dans 18 cas il avait été ajouté pour le vol à l'étude. Ce rajout apparaît un peu inutile quand on note que le chef est généralement retiré.

$\mathrm{Si}$ on prend en considération tous les chefs figurant dans la dénonciation, on évalue que $39,2 \%(40 / 102)$ des mineurs 
plaident coupable sur au moins un chef. An niveau adulte ces proportions étaient respectivement de $5,4 \%$ et de $83,3 \%$. A propos des chefs retirés $39,2 \%$ des jeunes ont au moins un chef de retiré contre $22,3 \%$ chez les adultes. Ces comparaisons avec les adultes sont sans grande importance puisqu'à partir du moment où le jeune est coupable sur au moins un chef, le juge est autorisé à adopter quelque mesure que ce soit.

Pour les huit cas où le jeune n'est coupable sur aucun chef, on note : cinq cas d'acquittement, et trois cas où tous les chefs sont retirés. La proportion des acquittements est donc très faible $(2,9 \%)$; au niveau adulte, cette proportion était de $2,5 \%$.

Cela signifie que pour tous les mineurs coupables sur au moins un chef, le juge peut décider d'une mesure parmi toutes celles prévues en vertu de la Loi sur les jeunes délinquants. Dans la section suivante, nous allons regarder de plus près les mesures et chercher à voir s'il existe un lien entre le choix de ces dernières et les caractéristiques propres au délit, à la dénonciation ainsi qu'au mineur lui-même.

\subsection{LES MESURES PRISES À L'ÉGARD DES MINEURS IMPLIQUÉS DANS UN VOL À MAIN ARMÉE}

Il reste donc 94 cas pour lesquels le juge décide d'une mesure. Le nombre de ces mesures est varié et le tribunal peut composer une mesure «personnalisée». Pour 62 jeunes, il n'y en a eu qu'une $(65,9 \%)$, pour 29 il y en a eu deux $(30,8 \%)$ et dans trois cas seulement il y en a eu trois. Il peut donc y avoir de multiples arrangements et nous les avons regroupés de manière à établir une certaine hiérarchie de sévérité (tableau 7).

Il y a un peu moins du quart des jeunes pour lesquels il y a hébergement sécuritaire, ce qui constitue une mesure parmi les plus sérieuses. Pour huit cas, il n'y a que cet hébergement comme mesure alors que pour six jeunes il est suivi d'une liberté surveillée et pour six autres d'un placement en centre d'accueil. La durée de l'hébergement varie de une à soixante-dix-huit semaines c'est-à-dire un an et demi. La moyenne se situe à 18 semaines, ce qui représente environ quatre mois et demi. Pour presque $30 \%$ des mineurs le juge a décidé d'un placement en centre d'accueil; la durée moyenne du placement est de 53,6 semaines avec une médiane de 52,5 , ce 


\section{TABLEAU 7}

Mesure imposée par le juge pour les mineurs impliqués dans au moins un vol à main armée

\begin{tabular}{llr} 
Mesure & $\mathrm{N}$ & $\%$ \\
\hline $\begin{array}{l}\text { Hébergement sécuritaire avec liberté surveillée } \\
\text { ou centre d'accueil }\end{array}$ & 21 & 22,3 \\
$\begin{array}{l}\text { Placement en centre d'accueil (pour un cas plus } \\
\text { travaux communautaires) }\end{array}$ & 28 & 29,8 \\
$\begin{array}{l}\text { Liberté surveillée (trois cas plus amende, } \\
\text { un cas plus restitution) }\end{array}$ & 23 & 24,5 \\
$\begin{array}{l}\text { Confié au D.P.J. et laissé à la garde } \\
\text { d'un parent ou les deux'1 }\end{array}$ & 11 & 11,7 \\
$\begin{array}{l}\text { Mesure autre que confié au D.P.J.2 } \\
\text { Total }\end{array}$ & 11 & 11,9 \\
\end{tabular}

1. Pour 8 de ces cas, il y a aussi de la liberté surveillée.

2. 2 cas : règlement définitif

5 cas : ajournement sine die, article 20

3 cas : amende

1 cas : amende et ordonnance de restitution

qui revient à dire que le jeune est généralement placé pour une période d'un an. Remarquons qu'il n'y a qu'un de ces cas pour lequel le placement est assorti d'une autre mesure qui est en l'occurrence une tâche de travail communautaire. Pour près du quart des jeunes $(24,5 \%)$, la mesure se limite à la liberté surveillée au service de probation; dans trois cas il y a également amende et pour un jeune il y a ordonnance de restitution. La durée de la surveillance le plus souvent est de un an (moyenne 45,5 semaines médiane 51,6$)$. Pour $11,6 \%$ des mineurs, le juge confie l'enfant à la Direction de la protection de la jeunesse pour qu'il soit laissé à la garde d'un parent (père, mère, oncle, tante, ...) ou des deux parents; huit de ces jeunes ont également eu une période de liberté surveillée d'une durée moyenne de 26,5 semaines avec une médiane de 12 semaines. Il semble donc que le jeune qui ne reçoit que de la probation doit se rapporter plus longtemps $(45,5$ semaines) que celui qui est aussi confié à ses parents $(26,5$ semaines). Enfin, il y a $11,7 \%$ des jeunes qui ne sont pas confiés à la Direction de la protection de la jeunesse pour l'application d'une quelconque mesure : pour cinq cas, le juge prononce un 
ajournement sine die, ce qui correspond à toute fin utile à une absence de mesure, pour deux cas, il $\mathrm{y}$ a un règlement définitif suspendu, pour trois cas on donne une amende dont le montant ne dépasse pas $50 \$(10 \$, 25 \$, 50 \$$, moyenne $=27,5)$.

En termes de sévérité, on peut penser que l'hébergement sécuritaire vient en premier lieu suivi du placement en centre d'accueil. À l'autre extrémité les jeunes qui ne sont pas confiés à la D.P.J. représentent les cas pour lesquels le juge a considéré qu'il n'y avait pas lieu de sévir; ceux qui sont confiés à leurs parents restent en liberté bien qu'il y ait un certain contrôle de la probation pour huit des onze cas. Ceux enfin qui ne sont l'objet que d'une mesure de probation peuvent être associés au groupe précédent en ce qui a trait au caractère contraignant de la mesure.

\subsubsection{Mesures selon le type de vol à main armée}

Si nous considérons le type de vol à main armée commis par le jeune et la mesure choisie par le juge, nous observons certaines différences intéressantes. Étant donné qu'il n'y a que trois jeunes ayant réalisé seul leur vol à main armée, nous avons procédé aux regroupements suivants : type 1 : institutions financières, groupe et seul; type 2 : commerces intermédiaires, groupe et seul; type 3 : variétés, dépanneurs, groupe et seul; type $4:$ particuliers, groupe et seul (voir tableau 8).

Les mineurs ayant volé une institution financière sont placés en centre d'accueil dans $50 \%$ des cas, ce qui constitue le pourcentage le plus élevé par rapport aux autres cibles. Ceux par ailleurs qui s'en prennent aux commerces intermédiaires sont envoyés en hébergement sécuritaire, notamment dans près de $40 \%$ des cas. ce qui est très élevé en comparaison des autres types de vol à main armée. Près du tiers de ceux qui choisissent les variétés et dépanneurs ne reçoivent pour toute mesure qu'une période de liberté surveillée au service de probation alors que $28,1 \%$ sont placés en centre d'accueil. Les vols à main armée commis contre les particuliers sont ceux où les mineurs sont le moins souvent confiés au D.P.J. pour l'application d'une quelconque mesure $(22,7 \%)$, c'est-à-dire ceux pour lesquels la justice a été la plus clémente. À l'opposé il faut voir cependant que pour $31,8 \%$ de ces jeunes il y eu un placement en centre d'accueil. 


\section{TABLEAU 8}

Mesure selon les types de vol à main armée

\begin{tabular}{|c|c|c|c|c|c|}
\hline Mesure1 & $\begin{array}{c}\text { Institu- } \\
\text { tions fi- } \\
\text { nancières }\end{array}$ & $\begin{array}{l}\text { Commer- } \\
\text { ces inter- } \\
\text { médiaires }\end{array}$ & $\begin{array}{l}\text { Variétés } \\
\text { dépan- } \\
\text { neurs }\end{array}$ & $\begin{array}{l}\text { Parti- } \\
\text { culiers }\end{array}$ & $\begin{array}{c}\text { Total } \\
21(22,3)\end{array}$ \\
\hline $\begin{array}{l}\text { Hébergement } \\
\text { sécuritaire }\end{array}$ & $1(8,3)$ & $11(39,2)$ & $5(15,6)$ & $4(18,2)$ & \\
\hline $\begin{array}{l}\text { Placement en } \\
\text { centre d'accueil }\end{array}$ & $6(50,0)$ & $6(21,4)$ & $9(28,1)$ & $7(31,8)$ & $28(29,8)$ \\
\hline $\begin{array}{l}\text { Liberté surveillée } \\
\text { (probation) }\end{array}$ & $3(25,0)$ & $6(21,4)$ & $6(31,2)$ & $4(18,2)$ & $23(24,5)$ \\
\hline $\begin{array}{l}\text { Confié au D.P.J. } \\
\text { et laissé a la } \\
\text { garde d'un parent } \\
\text { ou des deux }\end{array}$ & - & $3(10,7)$ & $6(18,7)$ & $2(9,1)$ & $11(11,7)$ \\
\hline $\begin{array}{l}\text { Autre que confié } \\
\text { au D.P.J. }\end{array}$ & $2(16,7)$ & $2(7,1)$ & $2(6,2)$ & $5(22,7)$ & $11(11,7)$ \\
\hline Total & $12(12,7)$ & $28(29,8)$ & $32(34,0)$ & $22(23,4)$ & $94(100)$ \\
\hline
\end{tabular}

1. Voir tableau 7 pour dêtails sur ces mesures.

Les différences que nous observons ici ne semblent pas traduire des tendances suivant lesquelles certains types de mesures correspondraient automatiquement à certains types de vols à main armée. En fait, nous croyons plutôt que pour chacun des genres de vols à main armée il y a différents profils types de mineurs et que les mesures sont ajustées à l'individu et non au délit. Nous pensons qu'il en va ainsi parce que pour chaque catégorie de vol les différentes mesures sont représentées bien que les pourcentages soient parfois variables.

3.4.2. Mesure selon les caractéristiques individuelles

Une première donnée intéressante à analyser ici est l'âge du jeune impliqué dans un vol à main armée. Faut-il penser que pour 
les plus vieux le juge fera preuve d'une plus grande sévérité et pour les jeunes d'une plus grande clémence?

La proportion des jeunes envoyés en hébergement sécuritaire va en croissant des plus jeunes aux plus vieux $(13,3,15,8,25,0$, 28,6 ), ce qui permet de penser qu'on hésite à avoir recours à une telle mesure pour les plus jeunes. Ces derniers sont toutefois placés en centre d'accueil dans $60 \%$ des cas, ce qui représente la proportion la plus élevée. Pour les plus vieux, la mesure de liberté surveillée est adoptée dans un pourcentage de $42,9 \%$ des cas et il en va de même pour ceux de seize ans $(42,1 \%)$. Les jeunes qui ont 17 ans ne sont pas confiés au D.P.J. dans une proportion de $21,9 \%$ et exception faite d'un autre cas ils sont les seuls à être l'objet de cette mesure.

TABLEAU 9

Mesure selon l'âge

\begin{tabular}{lccccc}
\hline Mesure1 & $14-15^{2}$ & 16 & 17 & 18 & Total \\
\hline $\begin{array}{l}\text { Hébergement } \\
\text { sécuritaire }\end{array}$ & $2(13,3)$ & $3(15,8)$ & $8(25,0)$ & $6(28,6)$ & $19(21,8)$ \\
$\begin{array}{l}\text { Placement en } \\
\text { centre d'accueil }\end{array}$ & $9(60,0)$ & $5(26,3)$ & $9(28,1)$ & $4(19,0)$ & $27(30,3)$ \\
$\begin{array}{l}\text { Libertée surveillée } \\
\text { (probation) }\end{array}$ & $1(6,6)$ & $9(42,1)$ & $5(15,6)$ & $9(42,9)$ & $23(26,4)$ \\
$\begin{array}{l}\text { Confié au D.P.J. } \\
\text { et laissé à la } \\
\text { garde d'un parent } \\
\text { ou les deux }\end{array}$ & $3(20,0)$ & $3(15,8)$ & $3(9,4)$ & $1(4,8)$ & $10(11,5)$ \\
$\begin{array}{l}\text { Autre que } \\
\text { confié au D.P.J. }\end{array}$ & -- & -- & $7(21,9)$ & $1(4,8)$ & $8(9,2)$ \\
$\begin{array}{c}\text { Total } \\
\text { Tar }\end{array}$ & $15(17,2)$ & $19(21,8)$ & $32(36,8)$ & $21(24,1)$ & $87(100)$ \\
& & & & & \\
\end{tabular}

1. Voir tableau 7 pour détails sur les mesures.

2. Nous avons regroupé ces deux âges puisqu'il n'y avait que trois sujets.

3. I1 y a 7 cas pour lesquels nous n'avons pas l'âge. 
Quant aux antécédents (tableaux 10 et 11), il ressort clairement de ces données que le jeune qui a déjà été signalé à la P.D.J. pour un motif de délinquance se voit l'objet d'une mesure plus contraignante puisque pour $66,5 \%$ des cas il y a placement contre

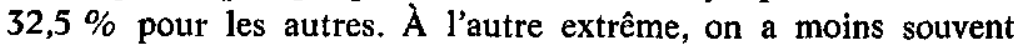
recours aux mesures non privatives de liberté, c'est-à-dire le retour au foyer, l'ajournement sine die ou encore l'amende. Il en va de même pour les signalements antérieurs pour motif de protection.

Pourquoi $74,9 \%$ de ceux qui ont déjà été rapportés à la D.P.J. pour cause de protection sont placés soit en hébergement sécuritaire, soit en centre d'accueil, ce pourcentage représente une proportion légalement supérieure à celle calculée chez les mineurs signalés pour délinquance $(66,6 \%)$. Ceux qui n'ont pas de motif antérieur de protection sont ainsi placés dans une proportion de $40,2 \%$. Que le motif en soit un de délinquance ou de protection n'a pas vraiment d'importance; le seul fait d'être connu de la D.P.J. favorise une mesure restrictive de liberté plus fréquente. Il y a 63 jeunes qui sont connus de la D.P.J., soit pour protection, soit pour délinquance et il y en a 31 qui n'ont jamais été signalés.

\section{TABLEAU 10}

Mesure selon la présence ou l'absence de signalement antérieur pour l'article 40 depuis janvier 1979

\begin{tabular}{lcc}
$\begin{array}{l}\text { Mesure } \\
\text { Sébergement sécuritaire }\end{array}$ & $\begin{array}{c}\text { Signalement antérieur pour délinquance } \\
\text { Non }\end{array}$ \\
\hline $\begin{array}{l}\text { Placement en centre d'accueil } \\
\text { Liberté surveillée (probation) }\end{array}$ & $24(44,4)$ & $9(22,5)$ \\
$\begin{array}{l}\text { Confié à D.P.J. et laissé à la garde } \\
\text { d'un parent ou des deux }\end{array}$ & $11(20,3)$ & $12(10,0)$ \\
$\begin{array}{l}\text { Autre que confié au D.P.J. } \\
\text { Total }\end{array}$ & $4(5,0)$ & $8(20,0)$ \\
& 54 & $7(17,5)$
\end{tabular}


TABLEAU 11

Mesure selon la présence ou l'absence de signalement antérieur pour l'article 38 depuis janvier 1979

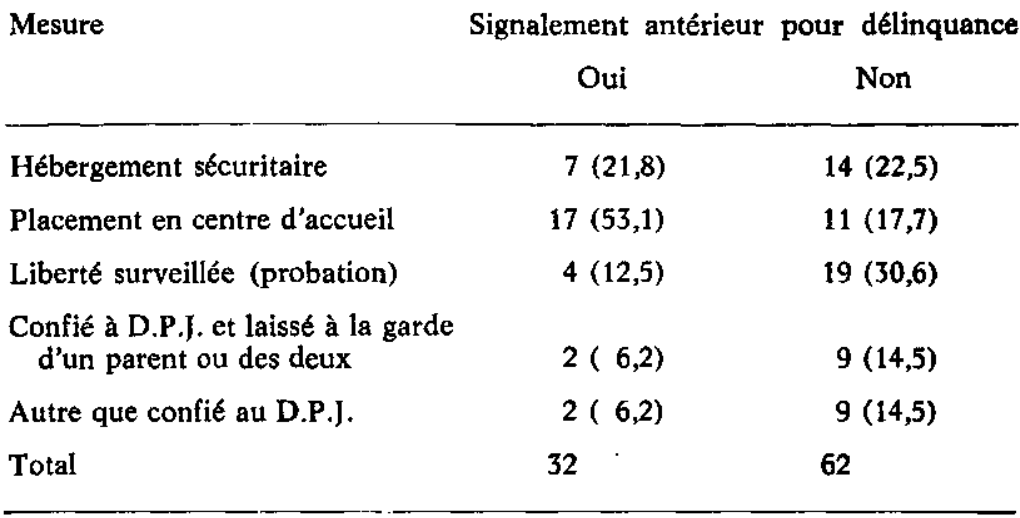

\section{FIGURE 1}

Mesure selon la présence ou l'absence de signalement antérieur à la D.P.J. depuis janvier 1979 (article 38 ou 40)

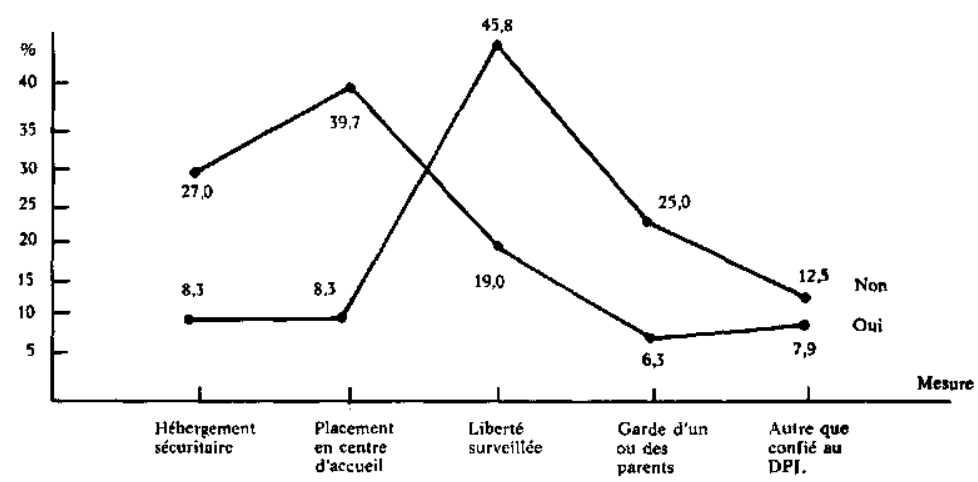

Les deux courbes de la figure 1 s'opposent suffisamment pour affirmer que le passé du jeune est largement pris en considération par le juge qui doit décider d'une mesure. Le fait d'avoir été déjà signalé à la D.P.J. favorise une mesure privative de liberté et à 
l'opposé, l'absence de tels signalements prédispose à des mesures en milieu libre. Tout se passe comme si, indépendamment de l'offense, le juge procédait par gradation dans ses tentatives : on essaye les mesures non contraignantes d'abord; après quoi, en cas de récidive, on passe aux mesures plus sévères. Cela se vérifie également par le fait que $55 \%$ de ceux qui ont déjà été placés en centre d'accueil le sont pour le vol à main armée de l'étude contre $19,9 \%$ pour ceux qui ne l'ont jamais été. Par ailleurs, $66,6 \%$ de ceux qui ont déjà comparu devant le tribunal avant janvier 1979 seront envoyés en centre d'accueil ou en hébergement sécuritaire contre $41,7 \%$ pour ceux qui n'ont jamais été devant le juge avant janvier 1979.

Ces derniers chiffres en disent long sur le processus décisionnel concernant le choix des mesures prises au tribunal de la jeunesse : le jeune est au cœur de leur décision. Des antécédents sociaux ou délictuels sont à l'origine de la plupart des placements et à l'inverse, quand le jeune n'a pas d'antécédent, il se trouve plus souvent en liberté.

\section{LES DÉLAIS DANS LE TRAITEMENT DES CAS CHEZ LES MINEURS}

La rapidité avec laquelle réagissent les instances officielles envers les mineurs ayant commis des délits peut être de nature, particulièrement chez les jeunes, à mettre en relief la relation de cause à effet entre le délit et la mesure. Bien que nous n'accordions plus à la mesure un caractère uniquement répressif et punitif, il faut voir que l'objectif ultime demeure toujours la dissuasion.

Entre le délit et la détection policière, il s'écoule en moyenne 33,2 jours, la médiane étant de 3,71 jours; entre le délit et le signalement, le délai moyen est de 47,9 jours avec une médiane de 9,17 (voir figure 1). A partir du moment où la D.P.J. reçoit le signalement, il se passe en moyenne 29,9 jours avant que la décision conjointe ne soit prise, le délai médian s'évaluant à huit jours. Cette dernière donnée rend compte de la rapidité avec laquelle on statue sur la question de judiciariser ou de ne pas judiciariser. En moyenne, il faut moins d'un mois, et pour $50 \%$ des cas, il faut huit jours ou moins. Lorsque le jeune est confié à un centre d'accueil pour plus de vingt-quatre heures sur des mesures d'urgence, il faut en moyenne 13,28 jours pour prendre la décision 
conjointe (médiane : 1,28) alors que ce délai est de 32,9 jours (médiane : 17,83) lorsque le jeune est en liberté. Au niveau de la D.P.J., donc, on rend une décision plus rapidement quand le jeune est privé de sa liberté. Au total, il s'écoule une moyenne de 75,7 jours entre le vol à main armée et la décision conjointe pour une médiane de 38,5 jours. Les moyennes sont ici influencées par quelques cas extrêmes et les médianes sont plus représentatives de la réalité.

Au niveau du tribunal de la jeunesse, nous apprenons qu'entre la première audition et la décision conjointe, il y a 22,4 jours en moyenne avec une médiane de six jours. Entre la première audition et le choix de la mesure, ces délais sont en moyenne de 102,6 jours et la médiane est de 52 jours. Lorsque le jeune n'est pas en libertê au cours des procédures, soit pour leur totalité soit en partie, le délai entre la première audition au tribunal et le choix de la mesure est de 47,2 jours en moyenne (médiane : 35,5 ) alors que pour les autres il est de 97,68 jours (médiane : 54,5). Il est donc manifeste que le tribunal procède plus rapidement que les cas des jeunes privés de liberté au cours des procédures. Au total, entre la décision conjointe et le choix de la mesure, il y a 125 jours en moyenne et 70,7 selon la médiane. Précisons que nous parlons ici de jours et non de jours ouvrables ce qui a pour effet de surévaluer les résultats ( 70 jours correspondent à 50 jours ouvrables). Quoiqu'il en soit, il est clair que pour $50 \%$ des mineurs, il faut compter deux mois et demi entre la décision conjointe et le choix de la mesure. Un tel délai est-il rapide ou trop long? Il n'est pas facile de répondre à cette question, il faut se donner le temps de connaître le jeune, c'est-à-dire de faire les évaluations qui s'imposent. Au niveau des adultes, nous avons vu que de la comparution à la sentence s'écoulait en moyenne 141,2 jours pour une médiane de 88,5 jours. Ce délai est légèrement supérieur à celui des mineurs : 102,6 jours entre la première audition et le choix de la mesure (médiane de 52). Au total, entre le délit et le choix de la mesure pour ceux qui sont judiciarisés, il s'écoule en moyenne de 181,92 jours avec une médiane de 134, c'est-à-dire d'environ quatre mois et demi.

\section{CONCLUSION}

La description est le point de départ de la connaissance, comme il n'existe pas de données dans les écrits sur la nature des 
vols à main armée chez les mineurs et leur cheminement dans le système de justice, des comparaisons et des conclusions sont donc difficiles, c'est pourquoi au terme de cet article il convient de rappeler les faits les plus significatifs.

\section{FIGURE 2}

Délais dans les procédures pour les mineurs (jours)

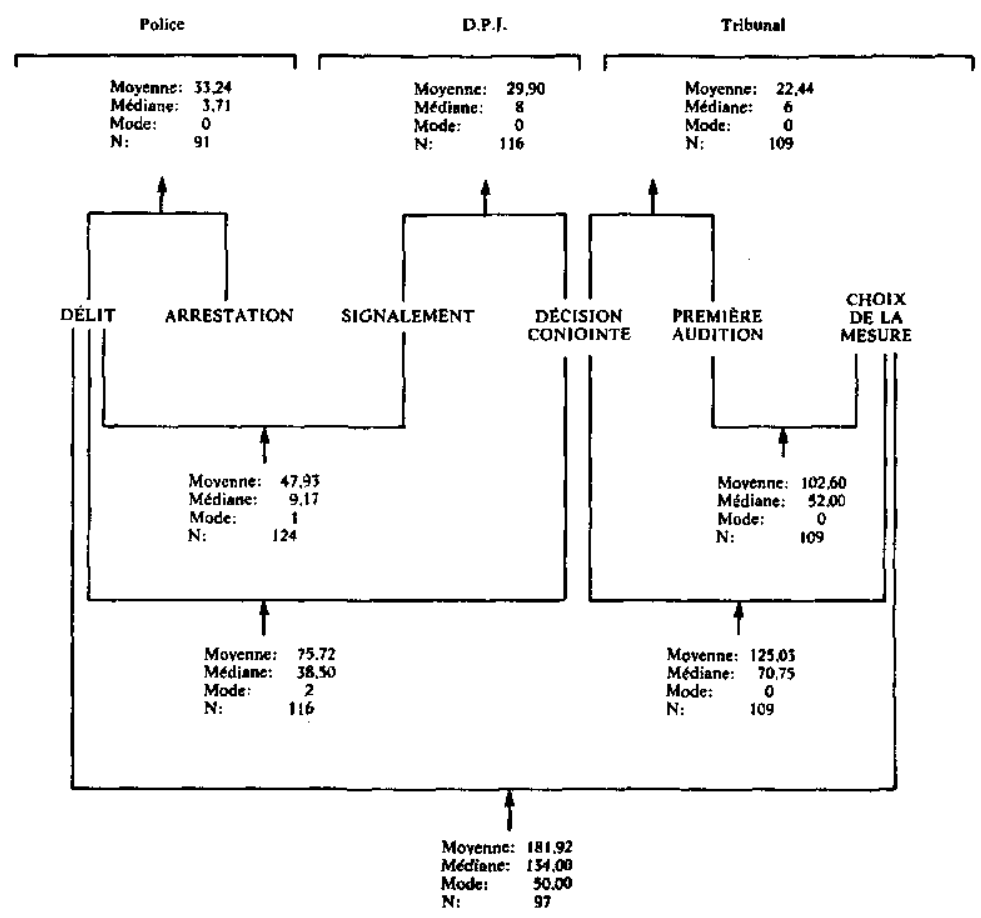

Les mineurs sont impliqués dans tous les types de vol à main armée exception faite de ceux qui sont commis seul. De plus, lorsqu'un jeune choisit de faire un vol à main armée, il privilégie les magasins de variétés, les dépanneurs et les particuliers dans $56 \%$ des cas contre $45,8 \%$ pour les adultes. Par ailleurs, plus souvent que ce n'est le cas pour les adultes, les policiers les poursuivent sans préciser l'alinéa de l'article 302 conférant par là le caractère équivoque de leur délit ou trahissant leur difficulté à 
préciser la nature d'une infraction commise par un mineur. Au niveau de la D.P.J., cela se reflète également puisque $26,4 \%$ des jeunes sont signalés pour un vol qualifié pour lequel on dit ignorer s'il y avait ou non une arme. Au niveau du tribunal, toutefois, ces nuances s'estompent, puisque les mineurs sont essentiellement poursuivis en vertu d'un article $302 \mathrm{D}$, le vol avec une arme.

Les signalements reçus à la D.P.J. sont chargés : pour $53,7 \%$ des mineurs plus d'un événement est rapporté. Cela laisse à penser que ces jeunes commettent une série de délits en peu de temps. L'étude détaillée de ces délits montre que le vol qualifié occupe une place de choix et que dans ce sens il y a une concentration dans cette forme de criminalité. Les mineurs signalés pour un vol à main armée sont connus pour avoir été signalés à la D.P.J. depuis janvier 1979 ou pour avoir comparu devant le tribunal avant cette date dans une proportion de $77,6 \%$. Le nombre des signalements antérieurs depuis janvier 1979 est impressionnant; il va de 1 à 24 et $28,8 \%$ des mineurs en ont six ou davantage. On observera, par ailleurs, que $44 \%$ de ces jeunes étaient connus de la cour de Bien-Être social, c'est-à-dire avant janvier 1979. Autre donnée intéressante : $60,8 \%$ de tous ces jeunes ont déjà été signalés à la D.P.J. pour un motif de délinquance et $38,4 \%$ pour un motif de protection (article 38). Ils ont en moyenne 16,6 ans et sont de sexe masculin. Ces quelques chiffres en disent long sur le passé des mineurs impliqués dans le vol à main armée : ils n'en sont pas à leur premier délit officiel et ils sont généralement fort connus des organismes sociaux.

Au niveau du tribunal, on constate d'abord que le nombre total des chefs d'accusation est impressionnant (1 à 47) avec une moyenne de 6,5 par jeune. Les délits commis ne sont donc pas «banalisés» au niveau du tribunal de la jeunesse. Comme cela se produit du côté adulte, on note que certains chefs sont retirés avec une certaine fréquence, notamment le recel, le complot et l'utilisation d'une arme à feu. Cela a toutefois moins d'impact qu'au niveau adulte en ce qui a trait à la mesure. D'une manière générale les jeunes plaident coupable et on observe que la proportion des acquittements est faible $(4,5 \%)$. Pour quatorze jeunes, il y a enquête en déféré et ce n'est que dans cinq cas que le jeune est envoyé devant le tribunal.

Les mesures prises à l'égard des jeunes vont de celles qui sont privatives de liberté aux autres qui s'exercent en milieu libre; 
l'ampleur des dénonciations pas plus que le type du vol à main armée ne semblent liés au choix de cette mesure. De manière très évidente c'est le passé du jeune qui s'avère déterminant, c'est-à-dire le fait d'avoir été signalé à la D.P.J. ou d'être passé devant le tribunal. On peut dire qu'à la D.P.J., la nature du délit compte pour beaucoup dans la décision de judiciariser en ceci qu'à peu près tous les jeunes impliqués dans un vol à main armée sont référés au tribunal. Au niveau de cette dernière instance, toutefois, le délit perd de son importance au profit des caractéristiques individuelles touchant aux signalements antérieurs. Tout se passe comme si la mesure gagnait en «sévérité» à partir du moment ou d'autres mesures antérieures s'étaient montrées inopérantes; on mise d'abord sur des mesures "clémentes» et en cas de récidive on exerce une plus grande contrainte sur le jeune.

L'ensemble de ces résultats confirment que l'adolescent constitue le centre de la décision que prend le juge : la gravité objective du délit n'a de sens que lorsque considérée simultanément avec les caractéristiques du jeune. Tout demeure toutefois lié en ceci que le vol à main armée ne constitue pas un premier délit et qu'il est le plus souvent la suite logique d'une série de délits. Malgré le fait que la justice pour mineurs n'est pas astreinte à des procédures plus formelles, la personne délinquante demeure toujours au centre de la décision et la gravité objective du délit est relayée au second plan contrairement à ce que l'on observe chez les adultes. Cette tendance fondamentale du système de justice persistera-t-elle sous la Loi sur les jeunes contrevenants?

\section{REFERENCES}

BELLOT, S., M. LEBLANC (1984), le Vol à main armée parmi l'activité délinquante, Recherche: Le vol à main armée, rapport technique no 10, Montréal, G.R.I.J./C.I.C.C. 58 p.

BRUNEAU, S., M.J. LAVIGUEUR, A. NORMANDEAU (1981), Le vol à main armée et les mineurs à Montréal, Apprentissage et socialisation, $4,2,181 \cdot 115$.

FRECHETTE, M., M. LEBLANC (1979), la Délinquance cachée d̀ l'adolescence, Montréal, G.R.I.J., Cahier du Groupe de recherche sur l'inadaptation juvénile, no $1,239 \mathrm{p}$.

FRECHETTE, M., M. LEBLANC (1985), la Délinquance à l'adolescence, Chicoutimi, Gaétan Morin.

GAGNON, R., M. LEBLANC (1984), le Vol à main armée dans les systèmes de justice, Recherche : Le vol à main armée, rapport final no 3, Montréal, G.R.I.J./C.I.C.C., 357 p. 